\title{
Functional analysis of the Frzb2 gene in Schistosoma japonicum
}

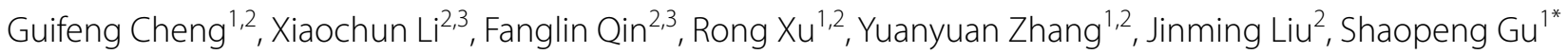 \\ and Yamei $\operatorname{Jin}^{2^{*}}$ (1)
}

\begin{abstract}
Schistosomiasis is a globally important helminthic disease of humans and animals, and it is the second most common parasitic disease after malaria. Eggs produced by mature females are responsible for the disease's occurrence and spread. Frzb2, a secreted frizzled-related protein, can inhibit Wnt signalling by competitive binding to the specific frizzled protein receptor. In this study, the complete gene sequence of SjFrzb2 was obtained by using 3'-rapid amplification of cDNA ends technology. SjFrzb2 transcript levels at different stages of S. japonicum maturation were evaluated by quantitative real-time RT-PCR analysis. SjFrzb2 was expressed at all developmental stages examined and exhibited the highest transcription level in 7-day-old worms, then gradually decreased during the growth and developmental stages to reach the lowest level at 18 days post-infection. SjFrzb2 gene expression was higher in female worms than in male worms and was significantly higher in female worms from a single-sex infection than in female worms from a bisexual infection. The functions of SjFrzb2 were explored via a small interfering RNA-based gene silencing approach and the soaking method. The results showed that SjFrzb2 gene knockdown impaired the growth and development of S. japonicum in mice, affecting not only the survival and morphological structure of the worms but also their reproductive ability and the viability of the produced eggs. Collectively, these observations imply that Frzb2 may be a novel target for the development of immuno- and/or small molecule-based therapeutics to control schistosomiasis fecundity and transmission.
\end{abstract}

\section{Introduction}

Schistosomiasis infects over 200 million people globally and is a major public health concern in more than 70 developing countries $[1,2]$. Furthermore, schistosomiasis can contribute to carcinogenesis and to higher susceptibility to other pathogens such as human immunodeficiency virus $[3,4]$. Schistosomiasis is also a concern for travellers and immigrants in nonendemic countries [5-7]. Three major species of schistosomes (Schistosoma mansoni, S. haematobium, and S. japonicum) infect humans, among which $S$. japonicum is the most prevalent in China, Indonesia, and the Philippines [8, 9]. Bisexually

\footnotetext{
*Correspondence: shpgu@163.com; yameijin@shvri.ac.cn

${ }^{1}$ College of Animal Science and Veterinary Medicine, Shanxi Agricultural University, Shanxi, China

${ }^{2}$ Key Laboratory of Animal Parasitology, Ministry of Agriculture of China, Shanghai Veterinary Research Institute, Chinese Academy of Agricultural Sciences, Shanghai, China

Full list of author information is available at the end of the article
}

infected female worms paired with males can continuously release several thousand eggs each day for many months or even years [10]. The eggs produced by mature female worms are important for parasitic transmission and disease pathogenesis. Female worms from single-sex infections (SF) are in a continually immature state and are normally unable to lay eggs [11], thereby inhibiting the occurrence and spread of schistosomiasis.

The regulation of various signalling pathways is essential for the growth and development of organisms. Signalling pathways transmit a variety of molecules, such as extracellular hormones, growth factors, and cytokines, which trigger a series of reactions that participate in many important biological processes. Wnt signalling is a highly conserved pathway that plays pivotal roles in embryonic development as well as energy metabolism and balance [12, 13]. Previous studies have indicated that the Wnt signalling pathway is critical for the normal development of the mammalian reproductive system,

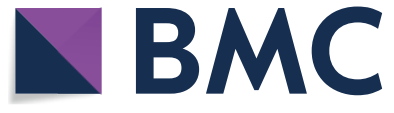

(c) The Author(s) 2019. This article is distributed under the terms of the Creative Commons Attribution 4.0 International License (http://creativecommons.org/licenses/by/4.0/), which permits unrestricted use, distribution, and reproduction in any medium, provided you give appropriate credit to the original author(s) and the source, provide a link to the Creative Commons license, and indicate if changes were made. The Creative Commons Public Domain Dedication waiver (http://creativecommons.org/ publicdomain/zero/1.0/) applies to the data made available in this article, unless otherwise stated. 
especially the formation of Müllerian ducts, and participates in the regulation of follicle development, ovulation, and luteinisation in the establishment of normal pregnancy [14-16]. Studies have also indicated that the inhibition of Wnt protein expression affects the reproductive development of $S$. japonicum.

The members of the secreted frizzled-related protein (sFrp) family can inhibit Wnt signalling by competitive binding to the specific frizzled protein receptor $(\mathrm{Fz}$ receptor) [17]. sFrp family members exist in humans, chickens, mice, and frogs (genus Xenopus) $[18,19]$. Frzb2 has been cloned in Xenopus and identified in the early anterior neural network of chickens. A previous study showed that ectopically expressed Frzb2 can inhibit head formation in Xenopus [20]. Moreover, a previous study by our group showed that the Frzb2 gene is highly expressed in SF compared with mature females from a bisexual infection (MF) (data not published), suggesting that SjFrzb2 is associated with the sexual maturity of female $S$. japonicum worms.

In the present study, the complete gene sequence of SjFrzb2 was obtained by 3'-rapid amplification of cDNA ends (RACE) technology. SjFrzb2 was analysed at the transcriptional level at different stages of $S$. japonicum maturity using small interfering RNA (siRNA) to explore the functional roles of SjFrzb2 in growth, development, and fecundity.

\section{Materials and methods}

\section{Ethics statement}

All animal experiments were conducted in accordance with the guidelines of the Committee for the Care and Use of Laboratory Animals of the Shanghai Veterinary Research Institute, Chinese Academy of Agricultural Sciences (permit no. SYXK-2016-0010). The study protocol was approved by the Ethics and Animal Welfare Committee of the Shanghai Veterinary Research Institute, Chinese Academy of Agricultural Sciences (experiment no. SHVRI-mo-2018040809).

\section{Parasites and animals}

The Chinese strain of S. japonicum used in this study was maintained at the National Institute of Parasitic Diseases, Chinese Center for Disease Control and Prevention. Freshly shed cercariae were obtained via the exposure of infected snails (Oncomelania hupensis) to light. Male BALB/c mice ( $n=60$; age, $4-6$ weeks; Shanghai SLAC laboratory Animal Co., Ltd., Shanghai, China) were percutaneously infected with bisexual or single-sex cercariae of S. japonicum. The worms were collected by hepatic-portal perfusion at different times, as described elsewhere [21]. The worms were then washed three times with phosphate-buffered saline (PBS, $\mathrm{pH}$ 7.4) at
$4{ }^{\circ} \mathrm{C}$ to remove residual host debris. The SFs were collected directly. Partial paired worms at $18,21,25,28,35$, and 42 days post-infection (dpi) were carefully separated from male and female worms (MF).

\section{Complete coding sequence (CDS) analysis}

The $3^{\prime}$-RACE primer GSP (5'-GAC TTC GAT GTT CCG GTT GTG TA-3') was designed with Primer 5.0 software (Premier Biosoft, Palo Alto, CA, USA) based on a SjFrzb2 reference sequence (GenBank accession no. AAX24900.2). Total RNA from 25 adult S. japonicum worms was extracted using TRIzol reagent (Invitrogen Corporation, Carlsbad, CA, USA) according to the manufacturer's instructions. The $3^{\prime}$-ends of the cDNA molecules were synthesized using the First Choice ${ }^{\circledR}$ RLM-RACE Kit (Invitrogen). The $3^{\prime}$ RACE-PCR products were purified and ligated into the pGEM T-easy vector (Takara Bio, Inc., Otsu, Shiga, Japan) and then transformed into competent Escherichia coli DH5 $\alpha$ cells (Tiangen Biotech (Beijing) Co., Ltd., Beijing, China). Clones were selected and subjected to DNA sequence analysis. The complete sequences along with the reference sequence were submitted to the GenBank database (accession number: MK253101). The complete sequences were analysed using the ORF Finder (Open Reading Frame Finder) graphical analysis tool [22]. The molecular weight, isoelectric point, and amino acid composition were determined using the ProtParam computational tool [23]. Signal peptide prediction was performed with the SignalP 3.0 server [24]. Transmembrane helices were analysed using the TMHMM method for the prediction of transmembrane protein topology based on a hidden Markov model [25]. Conserved domains were identified using the Conserved Domain Database [26]. The amino acid sequences of three orthologues were retrieved from the GenBank database for further multiple alignment analysis against the homologous sequences of Schistosoma mansoni, Mus musculus, and Homo sapiens using DNAMAN software.

\section{Quantitative real-time PCR analysis (qRT-PCR)}

Total RNA of S. japonicum at different stages was transcribed using the PrimeScriptTM ${ }^{\mathrm{RT}}$ reagent Kit (Takara Bio, Inc.). The following primers were used for qRT-PCR analysis: SjFrzb2-F (5'-TGA GAC GTC CAC CTT CAC AA-3')/SjFrzb2-R (5'-AGA CCG TCT AGT TGG TGT TG-3'), which amplified a 122-bp product of SjFrzb2; and SjTub-F (5'-ACC TCA ACA ACC ACC-3')/SjTub-R (5'TTG CGG CTT CTG CTC TTC-3'), which amplified a 234-bp product of the $\beta$-tubulin gene. qRT-PCR amplification was performed with the SYBR Premix Ex Taq ${ }^{\text {TM }}$ kit (Takara Bio, Inc.) and an ABI7500 Fast Real-Time PCR System (Applied Biosystems, Carlsbad, CA, USA). 
All experiments were performed in triplicate, and relative gene expression levels were calculated using the tubulin gene as an internal standard.

\section{Selection of effective siRNA of SjFrzb2}

Three specific siRNA pairs (SjFrzb2-S1, SjFrzb2-S2, and SjFrzb2- S3) were designed based on the SjFrzb2 gene sequence (GenBank accession no. MK253101) (Table 1). Each siRNA targeted a different region of the SjFrzb2 coding sequence. These siRNA pairs and a nonspecific (negative control, NC) siRNA pair (NC siRNA), with no homologous sequences in the schistosomal genome, were successfully used as control siRNA in other $S$. japonicum siRNA experiments [27-30] and were synthesized by Shanghai GenePharma Co., Ltd. (Shanghai, China). In this experiment, 10 mice were exposed to approximately 200 cercariae through shaved abdominal skin using the slide-cover-glass method and divided into five groups (2 mice/group). At day $22 \mathrm{dpi}$, the SjFrzb2 siRNAs and NC siRNA (dissolved in $200 \mu \mathrm{L}$ of diethyl pyrocarbonate-treated water) or PBS were rapidly injected via the tail vein. The final siRNA concentration adjusted to host blood was approximately $100 \mathrm{nM}$, and this concentration was confirmed to confer the greatest inhibitory effect against $S$. japonicum siRNA using the socking method [31]. After $48 \mathrm{~h}$, parasites were recovered from the hepatic veins by perfusion. Then, total RNA was isolated and amplified by qRT-PCR to evaluate interference effects.
siRNA for the long-term knockdown of SjFrzb2 gene expression

S1 siRNA was selected for long-term interference to evaluate the effects of SjFrzb2 on the parasitic development of S. japonicum. Twelve mice infected with $40 \pm 5$ cercariae were divided into three groups ( 4 mice/group). Long-term dose administration was used to maintain the siRNA concentration. Starting at $4 \mathrm{dpi}$, each mouse received 10 injections of 1 OD SjFrzb2-S1, NC siRNA (dissolved in $200 \mu \mathrm{L}$ of diethyl pyrocarbonate-treated water) or $200 \mu \mathrm{L}$ PBS via the tail vein every 4 days, as described in a previous study [32]. At $42 \mathrm{dpi}$, parasites were recovered and counted. The livers were also collected from the infected mice. Silencing effects were evaluated by qRT-PCR, electron microscopy, and calculation of the liver egg-hatching rate. qRT-PCR was also performed to detect the transcriptional levels of Wnt signalling-related genes [i.e., SjWnt1 (KM668879.1), SjWnt2 (DQ643830), SjWnt4 (DQ643829), and SjWnt5 (KC707587.1)] in worms after treatment with SjFrzb2-S1 siRNA. The primer sequences for the Wnt-related genes are shown in Table 2.

\section{Worm burden calculation, liver egg count, and miracidial hatching rate}

The worm burden reduction was calculated by comparing the number of worms recovered from the specific siRNA group to that of the control group using the formula WBR $=(1-$ WRSG/WRCG $) \times 100 \%$, where WRCG is the number of worms recovered from the control

Table 1 Sequences of SjFrzb2-specific siRNAs and the control siRNA

\begin{tabular}{lll}
\hline Name & Sequence & \\
\cline { 2 - 3 } & Sense & Anti-sense \\
\hline S1 siRNA & 5'-GCGCACAGAUUGCAUAUAUTT-3' & 5'-AUAUAUGCAAUCUGUGCGCTT-3' \\
S2 siRNA & 5'-GCUUCCAUCAACUAAUGUUTT-3' & 5'-AACAUUAGUUGAUGGAAGCTT-3' \\
S3 SiRNA & 5'-GGAGUAUUGCAUGUUGAAUTT-3' & 5'-AUUCAACAUGCAAUACUCCTT-3' \\
NC SiRNA & 5'-UUCUCCGAACGUGUCACGUTT-3' & 5'-ACGUGACACGUUCGGAGAATT-3' \\
\hline
\end{tabular}

*Reference sequence MK253101.

Table 2 Primer sequences for the amplification of Wnt signalling-related genes

\begin{tabular}{|c|c|c|c|}
\hline \multirow[t]{2}{*}{ Name } & \multicolumn{2}{|l|}{ Sequence } & \multirow{2}{*}{$\begin{array}{l}\text { Amplicon } \\
\text { length (bp) }\end{array}$} \\
\hline & Sense & Anti-sense & \\
\hline Sjwnt1 & 5'-ACAACGAAATCAACAACTTGCTCAC-3' & 5'-AAGTCAGTGGATGGGAATGTAGAAG-3’ & 106 \\
\hline Sjwnt2 & 5'-AATCGTGTAACCAAATGTAAATGCC-3' & 5'-CCAATCTTGGCTCATAAGTAACACG-3' & 125 \\
\hline Sjwnt4 & 5'-TATCATCAGTAGTATCAGGAGTATC-3' & 5'-TGGTGATGGTAAAGGCGATGTAGTC-3’ & 117 \\
\hline Sjwnt5 & 5'-ATAATAATAGAGCAGGTCGTTTGGC-3' & 5'-CTTGACGAAGATAACGACCAATACG-3' & 134 \\
\hline
\end{tabular}


group, and WRSG is the number of worms recovered from the siRNA group.

To evaluate the liver egg burden, the liver of each mouse was removed, weighed, homogenized, and digested with $10 \% \mathrm{NaOH}$ for $10 \mathrm{~min}$ at $56{ }^{\circ} \mathrm{C}$. The eggs were counted, and the number of liver eggs per gram was compared to that of the control mice using the formula $E R R=(1-E P G S G / E P G C G) \times 100 \%$, where ERR is the egg reduction rate, EPGCG is the number of eggs/g in the control group, and EPGSG is the number of eggs/g in the specific siRNA group.

To hatch the miracidia, $4 \mathrm{~mL}$ of liver homogenate was added to a flask filled with chlorine-free water. The neck of the flask was filled with a very thin layer of absorbent cotton, and the flask was incubated at $25-30{ }^{\circ} \mathrm{C}$ in the light. The supernatant above the cotton, which included the miracidia, was collected at $4 \mathrm{~h}$ after hatching. The miracidia were fixed with iodine and collected by centrifuging the supernatant at $4000 \times g$ for $5 \mathrm{~min}$ at $4{ }^{\circ} \mathrm{C}$. The numbers of miracidia were counted under a light microscope to calculate the hatching rates (i.e., number of observed miracidia divided by the number of added eggs). The hatching rate reduction was calculated by comparison to the control group using the formula $\mathrm{HRR}=(1-\mathrm{HRSG} / \mathrm{HRCG}) \times 100 \%$, where $\mathrm{HRR}$ is the hatching rate reduction, HRCG is the egg-hatching rate of the control group, and HRSG is the egg-hatching rate of the specific siRNA group.

\section{Scanning electron microscopy (SEM) and transmission electron microscopy (TEM)}

Adult worms were collected from the infected mice by perfusion and cleaned with PBS (pH 7.4), then fixed in $2 \%$ paraformaldehyde and $2.5 \%$ glutaraldehyde at $4{ }^{\circ} \mathrm{C}$ for $24 \mathrm{~h}$. For SEM, the samples were washed three times (15 min/time) with PBS, fixed in $1 \%$ osmium tetroxide for $2 \mathrm{~h}$, and then dehydrated in a graded ethanol series of $30 \%, 50 \%, 70 \%, 80 \%, 90 \%, 95 \%$, and $100 \%$ for $20 \mathrm{~min}$ each time. After desiccation in a $\mathrm{CO}_{2}$ critical point drying apparatus, the dehydrated worms were coated with a gold film in a vacuum-coating device and then examined under a JSM-6380LV scanning electron microscope (JEOL, Ltd., Tokyo, Japan). Micrographs were obtained at a magnification of $4000 \times$. For TEM, the samples were washed with PBS, dehydrated in $50 \%$ ethanol, $70 \%$ ethanol, $90 \%$ ethanol, $45 \%$ ethanol $/ 45 \%$ acetone, $90 \%$ acetone, and $100 \%$ acetone for $20 \mathrm{~min}$ each, and then soaked in pure acetone/embedding solution at a 3:1 dilution for $2 \mathrm{~h}$, a 1:1 dilution for $4 \mathrm{~h}$, and a 1:3 dilution overnight. The embedded specimens were cut into 60-100 nmthick ultrathin sections using an ultramicrotome (EM UC7; Leica Microsystems, Wetzlar, Germany) and then double stained with uranyl acetate and lead citrate.
Ultrastructural alterations were observed with a Tecnai G2 Spirit BioTWIN TEM system (FEI Electron Optics International B.V., Eindhoven, Netherlands).

\section{Statistical analysis}

Data are presented as the mean \pm standard deviation (SD). All statistical analyses were performed with Student's $t$ test or one-way analysis of variance. A probability $(p)$ value of $\leq 0.05$ was considered statistically significant.

\section{Results \\ Complete CDS and bioinformatic sequence analysis of SjFrzb2}

The combination of the $3^{\prime}$ RACE-PCR product with the reference sequence (GenBank AAX24900.2) resulted in an SjFrzb2 sequence with a total length of $1047 \mathrm{bp}$, which was submitted to the GenBank database under accession no. MK253101. The coding sequence of this gene consisted of $855 \mathrm{bp}$, encoding a protein comprising 284 amino acids with a molecular weight of approximately $36.5 \mathrm{kDa}$ and an isoelectric point of 5.13 . The sequence presented no signal peptide or transmembrane domain but did include a cysteine-rich Frizzled C-terminal domain and a conserved netrin module. A comparison of the amino acid sequences showed that the Frzb2 segment of S. japonicum shared $70 \%, 21 \%$, and $21 \%$ identity with its orthologues in S. mansoni, M. musculus, and H. sapiens, respectively (Figure 1).

\section{Frzb2 transcript levels in S. japonicum}

The transcript levels of SjFrzb2 were evaluated at different developmental time points, between the sexes, and at different maturation stages using qRT-PCR analysis. The following eight stages were examined: $7,14,18,21,25$, 28,35 , and 42 days. After 18 days, the female and male worms were separated for examination. Additionally, the transcript levels of SjFrzb2 at 25 days in SF and 25 days in MF were examined. Differences in transcript levels were observed by comparison with the housekeeping gene $\beta$-tubulin. All samples were run three times in triplicate, and Student's $t$-test and one-way analysis of variance were used to analyse the data.

The results demonstrated that the SjFrzb2 mRNA was expressed in all developmental stages examined and exhibited the highest transcription level in 7-day-old worms. Overall, RNA expression gradually declined to the lowest level at 18 days and then somewhat increased (Figure 2A). The results also suggested that transcript levels were higher in female worms than in male worms (Figure 2B). SjFrzb2 mRNA expression was significantly higher at 25 days in SF than at 25 days in MF (Figure 2C). 


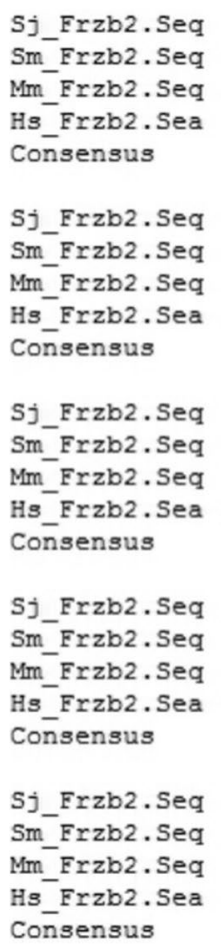

\section{Selection of an effective siRNA against SjFrzb2}

In the present study, the efficacy of three siRNAs (S1, S2, and S3) in the silencing of SjFrzb2 were first tested in vivo. At $22 \mathrm{dpi}$, the siRNAs were injected into infected mouse hosts ( $\sim 200$ cercariae) via the tail vein, and the parasites were harvested after $48 \mathrm{~h}$. The effects of gene silencing were measured by qRT-PCR. The median value of the NC siRNA group was set to $100 \%$, and the inhibition efficiency in the siRNA-treated groups was calculated as described previously [33]. The abundance of the SjFrzb2 gene transcript in the worms treated with the S1, S2, or S3 siRNA was reduced by $87.8 \%, 70.2 \%$, or $71 \%$, respectively, relative to the NC siRNA group (Figure $3 \mathrm{~A}$ ). Hence, the S1 siRNA was adopted to silence the expression of the SjFrzb2 transcript in the following long-term siRNA experiment.

\section{SjFrzb2 knockdown affects S. japonicum vitality, development, and reproduction}

For the long-term (42-day) siRNA experiment, infected mice were injected 10 times with SjFrzb2 S1 siRNA from 4 to 42 dpi every 4 days. Long-term treatment reduced SjFrzb2 transcript levels by $73 \%$ compared with the NC siRNA group (Figure 3B). SjFrzb2 silencing negatively affected parasitic vitality, development, and reproduction in mice. Compared with the NC and PBS groups, $45.95 \%$ and $35.49 \%$ of S. japonicum treated with SjFrzb2 in hosts did not survive to adulthood (Table 3). SjFrzb2 knockdown caused a decreases in the reproductive capacity of the surviving adult female worms of $33.28 \%$ and $46.70 \%$ compared with the NC and PBS groups, respectively. The loss of worms and reproductive capacity led to a lower liver egg burden. The liver egg burden reductions in the S1 siRNA group were $64.29 \%$ and $48.30 \%$, while $62.24 \%$ and $61.71 \%$ of liver eggs failed to hatch into miracidia compared with the NC and PBS groups, respectively.

\section{SjFrzb2 silencing leads to morphological changes in S. japonicum}

SjFrzb2 silencing also caused significant deformations of the adult worms. As shown in Figure 4, in comparison with the control siRNA group, the wall arrangement of females following SjFrzb2 siRNA treatment was disordered, and the wall protrusions were notably larger (Figures $4 \mathrm{~A}$ and $\mathrm{B}$ ). The bubbles were obviously increased, and the flower-like papillae exhibited abnormal structures (Figures $4 \mathrm{C}$ and $\mathrm{D}$ ). The appearance of the surfaces of the siRNA treated male worms were similar, with 

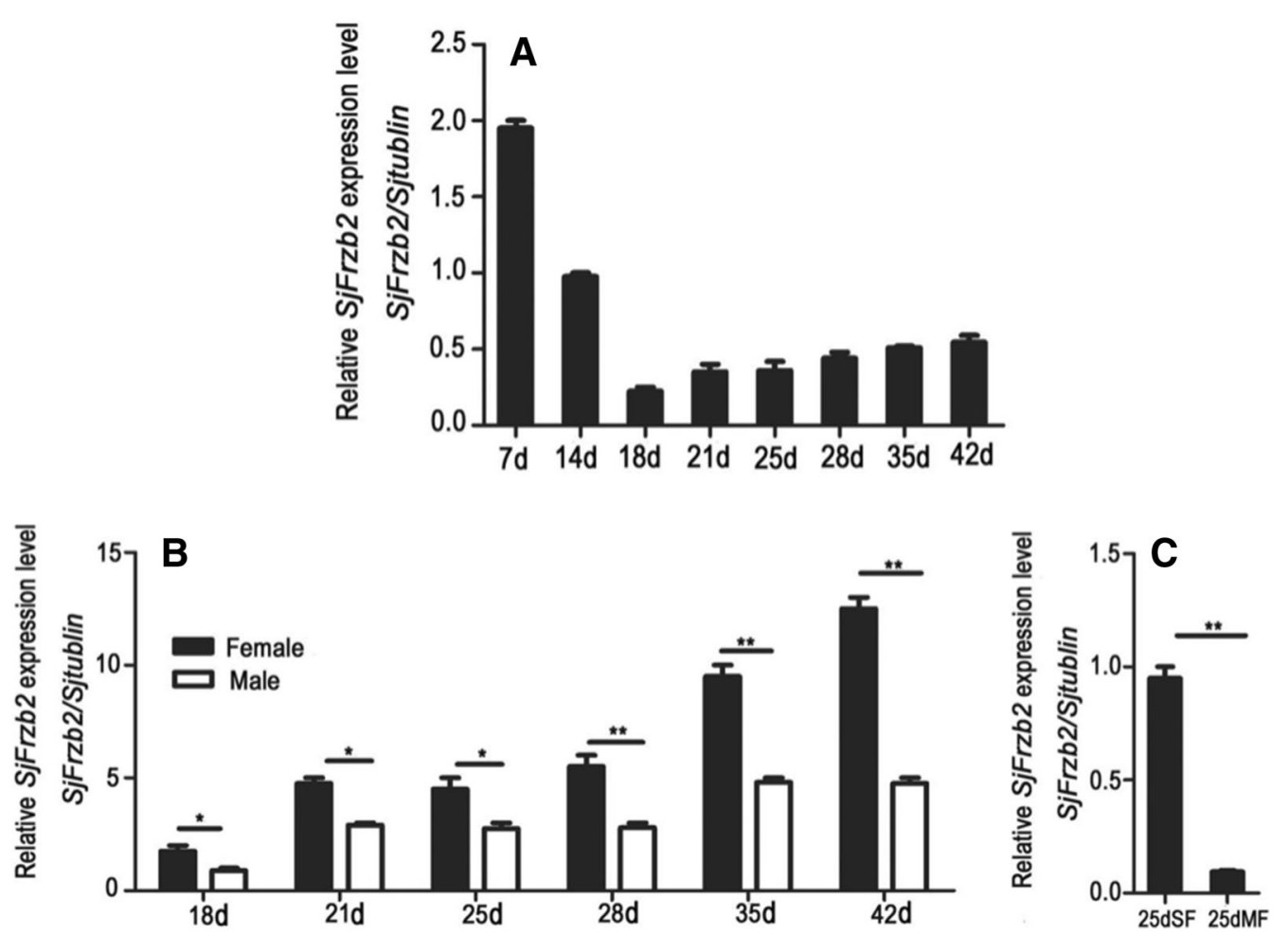

Figure 2 SjFrzb2 mRNA profiles detected by qRT-PCR. A A comparison of SjFrzb2 transcript levels at 7, 14, 18, 21, 25, 28, 35, and 42 dpi. B SjFrzb2 mRNA expression profiles in female worms and male worms at 18, 21, 25, 28, 35, and 42 dpi. C SjFrzb2 mRNA expression profile at 25 days (d) in SF (25-day-old female worms from a single-sex infection) and 25 days in MF (25-day-old female worms from a bisexual infection). S. japonicum $\beta$-tubulin was used as an internal control. All experiments were performed in triplicate, ${ }^{*} p<0.05,{ }^{* *} p<0.01 \mathrm{vs}$. the control group.

disordering of the network structure (Figures $4 \mathrm{E}$ and F) and obviously larger bubbles (Figures $4 \mathrm{G}$ and $\mathrm{H}$ ). In regard to the reproductive system, normally developed males presented greater numbers of spermatocytes, with chromatin around the nuclei, while the spermatocyte number was decreased, and the amount of intracellular chromatin was reduced in the siRNA-treated groups (Figures 5A and $\mathrm{B}$ ). The normally developed females exhibited mature vitelline cells with regular shapes of the cell edges and large numbers of vitelline droplets and globules, while females in the siRNA-treated groups exhibited developmentally immature vitelline cells with fewer vitelline droplets and Figures $5 \mathrm{C}-\mathrm{F}$.

\section{Effects of SjFrzb2 silencing on the transcript levels of Wnt signalling-related genes}

Because Frzb2 can inhibit Wnt signalling by competitive binding to the specific Fz receptor, a long-term interference experiment was conducted to detect the effect of SjFrzb2 silencing on Wnt signalling-related genes. Compared with the NC group, the expression levels of SjWnt1, SjWnt2, SjWnt4, and SjWnt5 were decreased by $50.24 \%, 70.16 \%, 59.88 \%$, and $42.65 \%$, respectively, as a result of SjFrzb2 gene interference (Figure 3C).

\section{Discussion}

The large number of eggs produced by mature female worms of S. japonicum is primarily responsible for the disease pathology and is critical for the dissemination of schistosomiasis [10]. During growth and development, the female worm undergoes very significant physiological, morphological, and immunoreactivity changes, including body wall transformations, cell differentiation, intestinal junction formation, and organogenesis [11]. Wnt signalling is critical during this process. The Frzb2 protein, as a part of the fine regulatory mechanism of Wnt signalling, plays a very important role in signal transmission [17]. The high expression of the SjFrzb2 protein in early stages, female worms, and SF worms (Figure 2) fully demonstrated its importance in the growth and development of $S$. japonicum, especially in females. Moreover, SjFrzb2 gene expression affects the expression patterns of other Wnt-related genes.

In schistosomes, the tegument is critical for nutritional uptake and signal transduction [34]. Since sexual maturation and spawning requires continuous male and female pairing, this interaction is necessary for the complete maturation of the vitelline gland and ovary and to ensure the normal development of egg cells and vitelline 

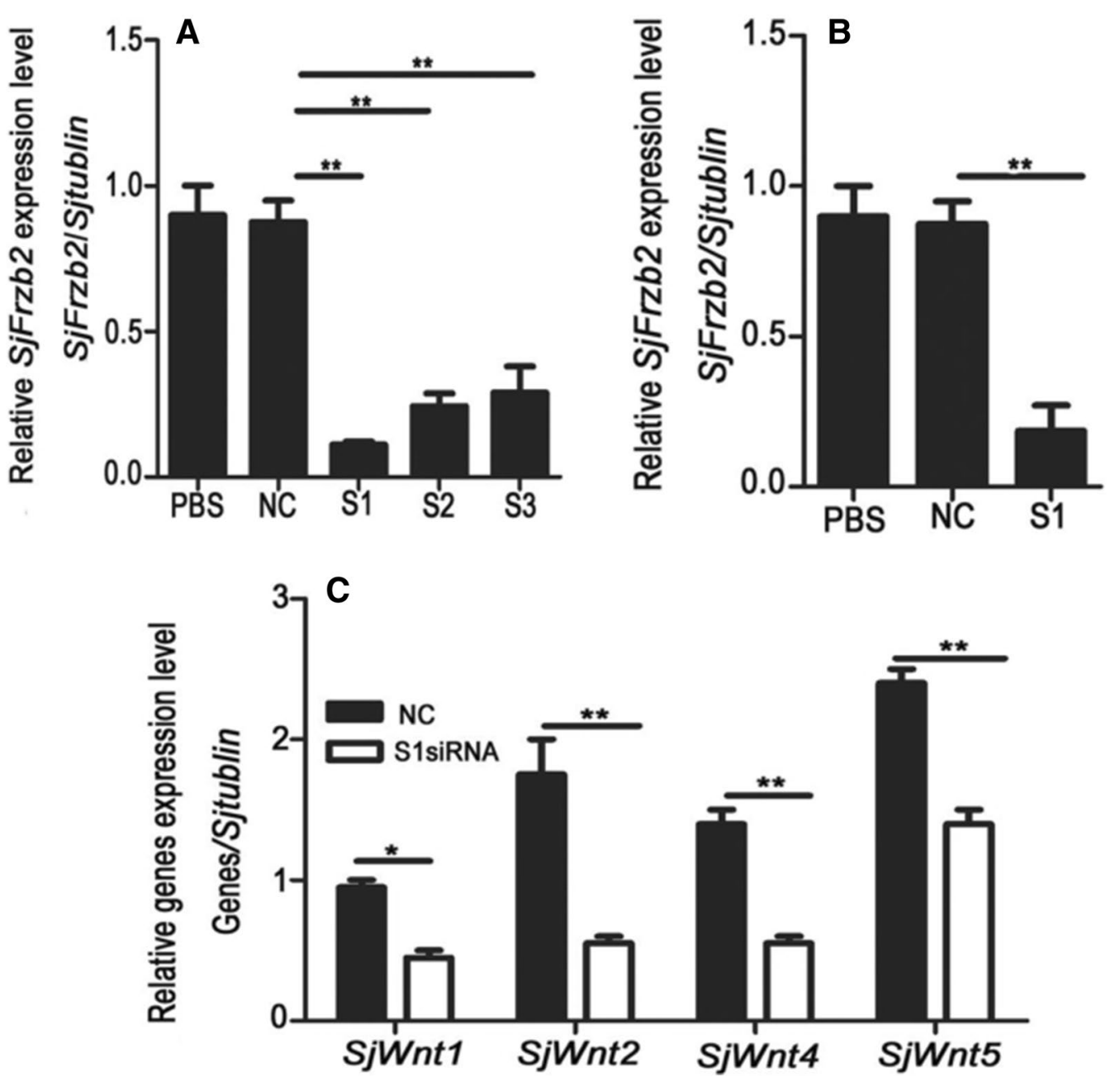

Figure 3 Effects of siRNA on SjFrzb2 expression normalized to $\beta$-tubulin mRNA. A Comparison of the efficacies of the NC and three SjFrzb2-specific siRNA species (S1, S2, and S3) on SjFrzb2 gene expression. B Effects of SjFrzb2-S1 siRNA on SjFrzb2 gene expression in adult worms after the SjFrzb2-S1 treatment of mice at all developmental stages (10 injections at 4-42 dpi). C Expression levels of Wnt signalling-related genes in SjFrzb2-interfered worms. Data are presented as the mean \pm SD of triplicate experiments. ${ }^{*} p<0.05,{ }^{* *} p<0.01$ vs. the control group.

Table 3 The effect of long-term SjFrzb2 interference on the vitality and fertility of S. japonicum

\begin{tabular}{lllll}
\hline Group $(\boldsymbol{n}=\mathbf{4})$ & Number of adults $(\mathbf{x} \pm \mathbf{s})$ & $\begin{array}{l}\text { Average egg burden/female } \\
\text { worm }(\mathbf{x} \pm \mathbf{s})\end{array}$ & $\begin{array}{l}\text { Average egg burden/gram } \\
\text { liver }(\mathbf{x} \pm \mathbf{s})\end{array}$ & Hatchability $(\mathbf{x} \pm \mathbf{s})$ \\
\hline PBS & $31 \pm 8.718$ & $1991 \pm 1014$ & $18,983 \pm 2250$ & $0.1954 \pm 0.0322$ \\
NC & $37 \pm 2.646$ & $1591 \pm 135$ & $27,484 \pm 784$ & $0.1981 \pm 0.0074$ \\
S1 & $20 \pm 2.449$ & $1061 \pm 190$ & $9814 \pm 2202$ & $0.0748 \pm 0.0098$ \\
$\%$ reduction & $35.49 \%^{* *}(\mathrm{PBS})$ & $46.70 \%^{* *}(\mathrm{PBS})$ & $48.30 \%^{* *}(\mathrm{PBS})$ & $61.71 \%^{* *}(\mathrm{PBS})$ \\
& $45.95 \%^{* *}(\mathrm{NC})$ & $33.28 \%^{* *}(\mathrm{NC})$ & $64.29 \%^{* *}(\mathrm{NC})$ & $62.24 \%^{* *}(\mathrm{NC})$ \\
\hline
\end{tabular}

** $p<0.01$.

cells. Abnormal tegument structures may affect information transmission between males and females and inhibit the normal absorption of glucose and amino acids. In the present study, SjFrzb2 knockdown not only caused significant morphological changes in the tegumental structure and gonadal cells of the parasites subjected to RNA interference (Figures 4,5) but also reduced the survival and fecundity of the worms, which, in turn, reduced 


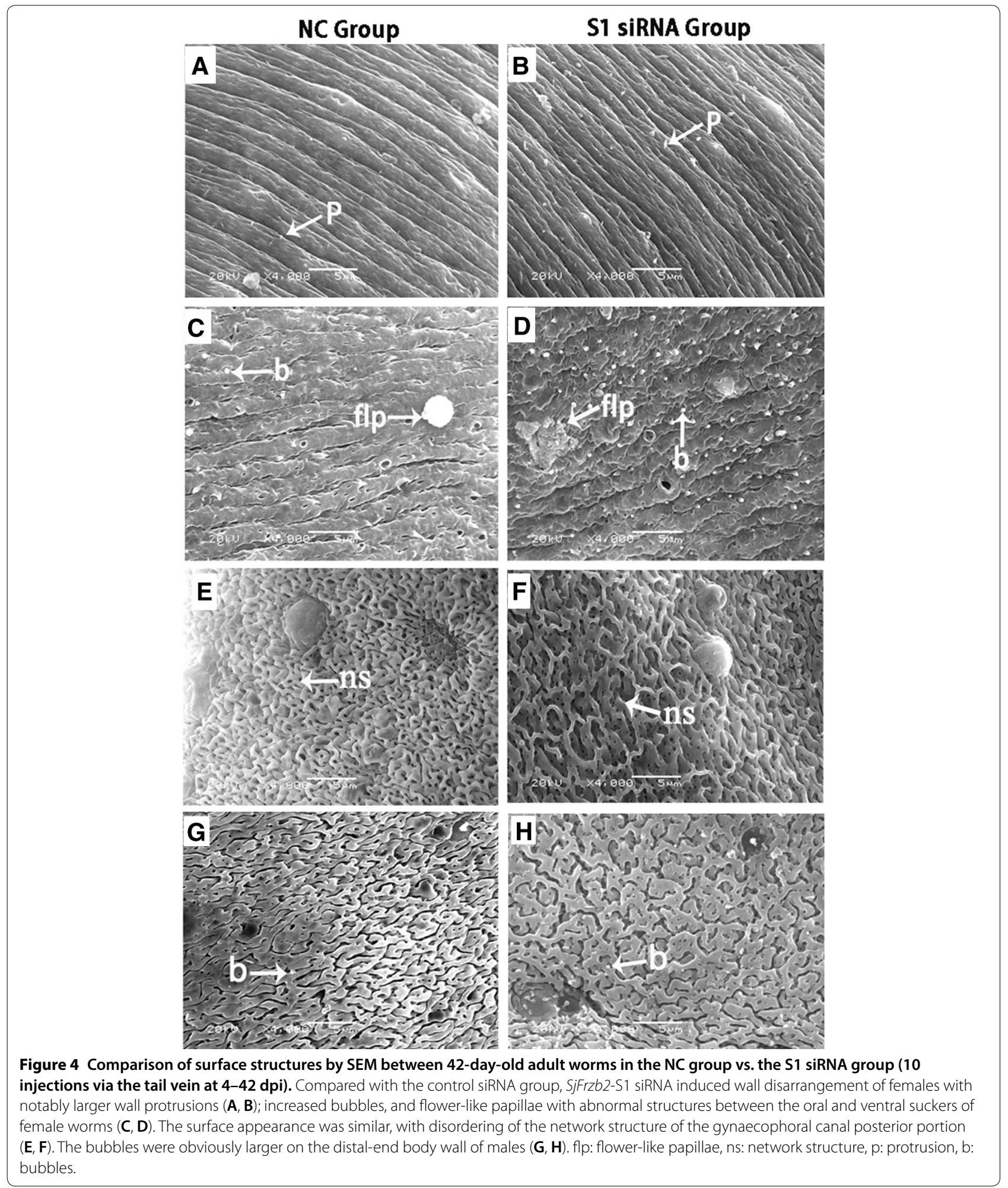

the host liver egg burden and egg viability (Table 3 ). Collectively, these effects contributed to the inhibition of the host pathological response and, subsequently, disease transmission.
In schistosomiasis, a vaccine that induces even a partial reduction in the worm burden could considerably reduce the pathology and limit parasitic transmission. Many potential vaccine antigens have been evaluated, 


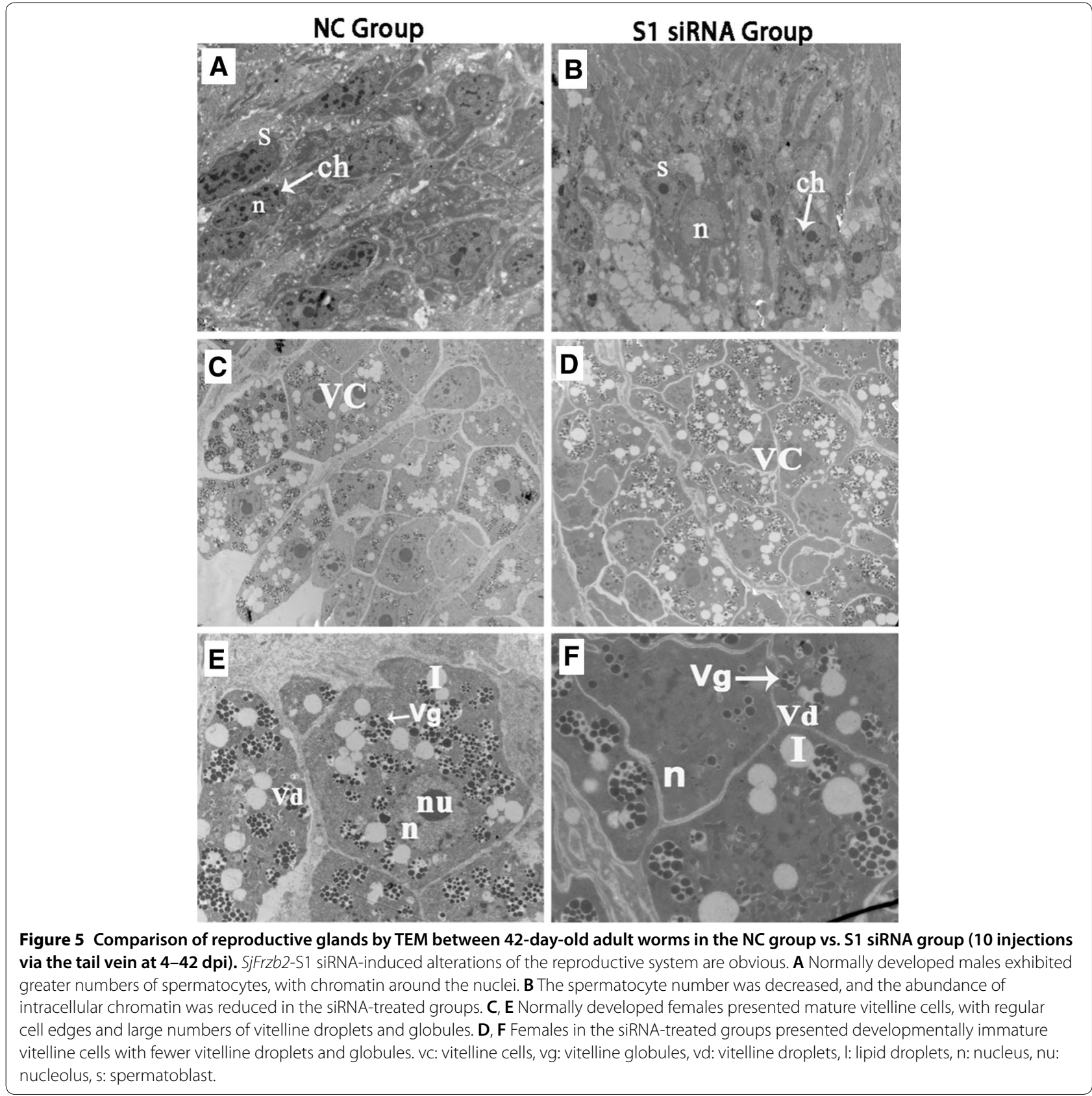

but none have demonstrated satisfactory protection when used in the clinic [35]. The level of protection obtained by vaccination with these antigens rarely exceeded the $40 \%$ benchmark set by the World Health Organization. At present, there is no commercial vaccine available, even though several candidate antigens have been suggested by the World Health Organization or reported by researchers. The best long-term strategy for controlling schistosomiasis is through immunization with an anti-schistosomiasis vaccine combined with drug treatment. SjFrzb2 is important for the growth, development, and reproduction of $S$. japonicum, as indicated not only by its mRNA expression profile, which is high in early schistosomules that are physiologically active, but also by the long-term siRNA experiment, in which the parasites subjected to RNA interference exhibited reductions in survival, fecundity, the live egg load, and egg-hatching rates. Based on these results, 
we propose that SjFrzb2 should be further explored as a novel target for the development of immuno- and/or small molecule-based therapeutics to control schistosomiasis fecundity and transmission.

\section{Acknowledgements}

This work was supported by grants from the Chinese National Natural Science Foundation (No. 31672245) and the Natural Science Foundation of Shanghai (No. 16ZR1444100). The authors thank International Science Editing for revising the manuscript and for their valuable comments.

\section{Authors' contributions}

GC: overall experimental design and execution of all laboratory experiments, writing of the manuscript, interpretation of all data; $X \mathrm{~L}, F Q, R X, Y Z$ : participated in the qRT-PCR analyses; JL: assisted with the siRNA experiments; SG, YJ: obtained financial support, conceived and designed the study and analysed the data. All authors read and approved the final manuscript.

\section{Funding}

This work was supported by grants from the Chinese National Natural Science Foundation (No. 31672245) and the Natural Science Foundation of Shanghai (No. 16ZR1444100).

\section{Competing interests}

The authors declare that they have no competing interests.

\section{Author details}

${ }^{1}$ College of Animal Science and Veterinary Medicine, Shanxi Agricultural University, Shanxi, China. ${ }^{2}$ Key Laboratory of Animal Parasitology, Ministry of Agriculture of China, Shanghai Veterinary Research Institute, Chinese Academy of Agricultural Sciences, Shanghai, China. ${ }^{3}$ College of Life Sciences, Shanghai Normal University, Shanghai, China.

Received: 18 June 2019 Accepted: 9 October 2019

Published online: 11 December 2019

\section{References}

1. Steinmann P, Keiser J, Bos R, Tanner M, Utzinger J (2006) Schistosomiasis and water resources development: systematic review, meta-analysis, and estimates of people at risk. Lancet Infect Dis 6:411-425

2. Weerakoon KG, Gobert GN, Cai P, McManus DP (2015) Advances in the diagnosis of human schistosomiasis. Clin Microbiol Rev 28:939-967

3. Correia da Costa JM, Vale N, Gouveia MJ, Botelho MC, Sripa B, Santos LL, Santos JH, Rinaldi G, Brindley PJ (2014) Schistosome and liver fluke derived catechol-estrogens and helminth associated cancers. Front Genet 5:444

4. Secor WE (2012) The effects of schistosomiasis on HIV/AIDS infection, progression and transmission. Curr Opin HIV AIDS 7:254-259

5. Grobusch MP, Mühlberger N, Jelinek T, Bisoffi Z, Corachán M, Harms G, Matteelli A, Fry G, Hatz C, Gjørup I, Schmid ML, Knobloch J, Puente S, Bronner U, Kapaun A, Clerinx J, Nielsen LN, Fleischer K, Beran J, da Cunha S, Schulze M, Myrvang B, Hellgren U (2003) Imported schistosomiasis in Europe: sentinel surveillance data from TropNetEurop. J Travel Med 10:164-169

6. Hatz CF (2005) Schistosomiasis: an underestimated problem in industrialized countries? J Travel Med 12:1-2

7. Beltrame A, Guerriero M, Angheben A, Gobbi F, Requena-Mendez A, Zammarchi L, Formenti F, Perandin F, Buonfrate D, Bisoffi Z (2017) Accuracy of parasitological and immunological tests for the screening of human schistosomiasis in immigrants and refugees from African countries: an approach with Latent Class Analysis. PLoS NegI Trop Dis 11:e0005593

8. Gryseels B, Polman K, Clerinx J, Kestens L (2006) Human schistosomiasis. Lancet 368:1106-1118

9. Hope M, Duke M, Mcmanus DP (1996) A biological and immunological comparison of Chinese and Philippine Schistosoma japonicum. Int J Parasitol 26:325-332
10. Shen J, Lai DH, Wilson RA, Chen YF, Wang LF, Yu ZL, Li MY, He P, Hide G, Sun $X$ (2017) Nitric oxide blocks the development of the human parasite Schistosoma japonicum. Proc Natl Acad Sci USA 114:10214-10219

11. Loverde PT, Chen L (1991) Schistosome female reproductive development. Parasitol Today 7:303-308

12. Nusse R (2015) Cell signalling: disarming Wnt. Nature 519:163-164

13. Shi J, Chi S, Xue J, Yang J, Li F, Liu X (2016) Emerging role and therapeutic implication of Wnt signaling pathways in autoimmune diseases. J Immunol Res 2016:9392132

14. Parr BA, Mcmahon AP (1998) Sexually dimorphic development of the mammalian reproductive tract requires Wnt-7a. Nature 395:707-710

15. Hsieh M (2002) Regulated expression of Wnts and Frizzleds at specific stages of follicular development in the rodent ovary. Endocrinology 143:898-908

16. Ricken A, Lochhead P, Kontogiannea M, Farookhi R (2002) Wnt signaling in the ovary: identification and compartmentalized expression of wnt-2 wnt-2b, and frizzled-4 mRNAs. Endocrinology 143:2741-2749

17. Ladher RK, Church VL, Allen S, Robson L, Abdelfattah A, Brown NA, Hattersley G, Rosen V, Luyten FP, Dale L, Francis-West PH (2000) Cloning and expression of the wnt antagonists Sfrp-2 and Frzb during chick development. Dev Biol 218:183-198

18. Stoehr R, Wissmann C, Suzuki H, Knuechel R, Krieg RC, Klopocki E, Dahl E, Wild P, Blaszyk H, Sauter G, Simon R, Schmitt R, Zaak D, Hofstaedter F, Rosenthal A, Baylin SB, Pilarsky C, Hartmann A (2004) Deletions of chromosome $8 p$ and loss of sFRP1 expression are progression markers of papillary bladder cancer. Lab Invest 84:465-478

19. Lee JL, Chang CJ, Wu SY, Sargan DR, Lin CT (2004) Secreted frizzledrelated protein 2 (SFRP2) is highly expressed in canine mammary gland tumors but not in normal mammary glands. Breast Cancer Res Treat 84:139-149

20. Bradley L, Sun B, Collins-Racie L, LaVallie E, McCoy J, Sive H (2000) Different activities of the frizzled-related proteins frzb2 and sizzled2 during Xenopus anteroposterior patterning. Dev Biol 227:118-132

21. Smithers SR, Terry RJ (1965) the infection of laboratory hosts with cercariae of Schistosoma mansoni and the recovery of the adult worms. Parasitology 55:695-700

22. ORF Finder Tool. https://www.ncbi.nlm.nih.gov/orffinder/. Accessed 20 Dec 2018

23. ProtParam Tool. http://web.expasy.org/protparam/. Accessed 20 Dec 2018

24. SignalP Tool. http://www.cbs.dtu.dk/services/SignalP/. Accessed 20 Dec 2018

25. TMHMM Tool. http://www.cbs.dtu.dk/services/TMHMM-2.0/. Accessed 20 Dec 2018

26. Conserved Domain Database. https://www.ncbi.nlm.nih.gov/cdd. Accessed 20 Dec 2018

27. Xu R, Zhang YY, Li XC, Cheng GF, He CC, Guo L, Li H, Liu JM, Gu SP, Jin YM (2018) Cloning, expression and function analysis of Schistosoma japonicum ELAV-like 1. Sci Agric Sin 51:2600-2613

28. Cao YF, Shi YL, Qiao HB, Yang YX, Liu JM, Shi YJ, Lin JJ, Zhu G, Jin YM (2014) Distribution of lethal giant larvae (Lgl) protein in the tegument and negative impact of siRNA-based gene silencing on worm surface structure and egg hatching in Schistosoma japonicum. Parasitol Res 113:1-9

29. Yang YX, Jin YM, Liu PP, Shi YL, Cao YF, Liu JM, Shi YJ, Li H, Lin JJ (2012) RNAi silencing of type $V$ collagen in Schistosoma japonicum affects parasite morphology, spawning, and hatching. Parasitol Res 111:251-1257

30. Zou X, Jin YM, Liu PP, Wu QJ, Liu M, Lin JJ (2011) RNAi silencing of calciumregulated heat-stable protein of $24 \mathrm{kDa}$ in Schistosoma japonicum affects parasite growth. Parasitol Res 108:567-572

31. Cheng GF, Lin JJ, Shi Y, Jin YX, Fu ZQ, Jin YA, Zhou YC, Cai YM (2005) Dosedependent inhibition of gynecophoral canal protein gene expression in vitro in the schistosome (Schistosoma japonicum) by RNA interference. Acta Biochim Biophys Sin 37:386-390

32. Cheng GF, Fu ZQ, Lin JJ, Shi Y, Zhou YC, Jin YX, Cai YM (2009) In vitro and in vivo evaluation of small interference RNA-mediated gynaecophoral canal protein silencing in Schistosoma japonicum. J Gene Med 11:412-421

33. Pfarr K, Heider U, Hoerauf A (2006) RNAi mediated silencing of actin expression in adult Litomosoides sigmodontis is specific, persistent and results in a phenotype. Int J Parasitol 36:661-669 
34. Van Hellemond JJ, Retra K, Brouwers JF, van Balkom BW, Yazdanbakhsh M, Shoemaker CB, Tielens AG (2006) Functions of the tegument of schistosomes: clues from the proteome and lipidome. Int J Parasitol 36:691-699

35. Bergquist NR, Colleyb DG (1998) Schistosomiasis vaccine: research to development. Parasitol Today 14:99-104

\section{Publisher's Note}

Springer Nature remains neutral with regard to jurisdictional claims in published maps and institutional affiliations.
Ready to submit your research? Choose BMC and benefit from:

- fast, convenient online submission

- thorough peer review by experienced researchers in your field

- rapid publication on acceptance

- support for research data, including large and complex data types

- gold Open Access which fosters wider collaboration and increased citations

- maximum visibility for your research: over $100 \mathrm{M}$ website views per year

At BMC, research is always in progress.

Learn more biomedcentral.com/submissions 